\title{
STRUCTURE THEORY OF SIMPLE RINGS WITHOUT FINITENESS ASSUMPTIONS
}

\author{
BY \\ N. JACOBSON
}

It is the purpose of this paper to lay the foundations of a general theory of simple rings, both associative and non-associative. In part I we obtain the structure of simple associative rings that either contain minimal right ideals or contain maximal right ideals. In either case we obtain a realization of our ring $\mathfrak{A}$ as a certain type of ring of linear transformations in a vector space over a division ring. If $\mathfrak{A}$ has a minimal right ideal this realization is essentially unique and we can prove a converse theorem that rings of linear transformations having certain properties are simple and contain minimal right ideals. Thus the theory here is essentially as complete as that of the classical case of rings that satisfy the descending chain condition for right ideals. Our structure theory hinges on a general theorem (Theorem 6 ) on the structure of irreducible rings of endomorphisms of commutative groups. This theorem may be regarded as an extension of Burnside's theorem on irreducible algebras of matrices. The classical theory of simple rings with descending chain condition is a simple consequence of our results and we believe that the present treatment is more transparent than the methods of proof previously given( ${ }^{(1)}$.

In studying an arbitrary non-associative ring $\mathfrak{A}$ one is led to consider the associative ring $\mathfrak{M}$ generated by the left and the right multiplications $x \rightarrow a x$ and $x \rightarrow x a$ acting in $\mathfrak{A}$. If $\mathfrak{A}$ is simple, $\mathfrak{M}$ is an irreducible ring of endomorphisms. In part II we describe the structure of $\mathfrak{M}$ in terms of the multiplication centralizer $\mathfrak{E}$ of $\mathfrak{A}$ defined to be the totality of endomorphisms $\boldsymbol{\gamma}$ in $\mathfrak{A}$ such that $(x y) \gamma=(x \gamma) y=x(y \gamma)$. We define the concepts of center, central algebra and extension of the underlying field of an algebra.

In Part III we investigate a special type of simple associative algebra that may be regarded as a generalization of the concept of the complete algebra of linear transformations in a vector space over a field, or equivalently, of the concept of the complete matrix algebra. There are many points of contact between the discussion here (and in other parts of the paper) and recent work in the theory of rings of transformations in Banach spaces and in vector spaces over the fields of real and complex numbers $\left({ }^{2}\right)$.

Presented to the Society, August 12,1944; received by the editors June 24, 1944.

(1) Cf. Jacobson [1, chap. 4] and Artin and Whaples [1]. Numbers in brackets refer to the Bibliography at the end of the paper.

(2) Cf. Arnold [1] and the references given in this paper. 


\section{STRUCTURE OF SIMPLE RINGS}

1. Dense rings of linear transformations. Let $\Re$ be an arbitrary vector space over a division ring $\mathfrak{D}$. We recall the definition: $\Re$ is a commutative group written additively and there is singled out a division ring $\mathfrak{D}$ of endomorphisms $\alpha, \beta, \ldots$ acting in $\Re$ that includes the identity transformation. We make no assumption as to the dimensionality of $\Re$ over $\mathfrak{D}$. Let $\mathfrak{R}$ be the ring of linear transformations (1.t.) in $\Re$ over $\mathfrak{D}$. Thus $\mathfrak{R}$ is the totality of endomorphisms in $\Re$ that commute with every $\alpha \in \mathfrak{D}$.

Let $\mathfrak{A}$ be a set of 1.t. Then we shall call $\mathfrak{A} k$-fold transitive if for any two ordered sets of vectors $x_{1}, \cdots, x_{k} ; y_{1}, \cdots, y_{k}$ such that the $x$ 's are linearly independent, there exists an $A \in \mathfrak{A}$ such that $x_{i} A=y_{i}, i=1, \cdots, k$. It is obvious that if $\mathfrak{A}$ is $k$-fold transitive, then $\mathfrak{A}$ is $l$-fold transitive for every $l<k$ and it is easy to see that if $\mathfrak{A}$ is $n$-fold transitive and the dimensionality $(\mathfrak{R}: \mathfrak{D})=n$, then $\mathfrak{A}=\mathfrak{R}$. If $\mathfrak{A}$ is 1 -fold transitive, $\mathfrak{A}$ is an irreducible set of endomorphisms in the sense that the only subgroups of $\Re$ invariant under every $A \in \mathfrak{A}$ are $\Re$ and 0 . Conversely if $\mathfrak{A} \neq 0$ is a subring of $\mathfrak{R}$ and is an irreducible set of endomorphisms, then $\mathfrak{A}$ is 1 -fold transitive. For if $x \neq 0$ is in $\Re$ the totality $x \mathfrak{A}$ of vectors $x A, A \in \mathfrak{A}$, is a subgroup invariant under $\mathfrak{A}$. If $x \mathfrak{A}=0$ the space $\{x\}$ of multiples $x \alpha$ is invariant and not equal to 0 . It follows that $\{x\}=\Re$ and this contradicts $\mathfrak{A} \neq 0$. Hence $x \mathfrak{A}=\Re$ and any $y$ in $\Re$ has the form $x A$ for a suitable $A$ in $\mathfrak{A}$.

If $\mathfrak{A}$ is a set of 1.t. that is $k$-fold transitive for each $k=1,2,3, \cdots$, then we shall say that $\mathfrak{A}$ is dense (in $\mathfrak{R}$ ). If $\Re$ is finite-dimensional and $\mathfrak{A}$ is dense, $\mathfrak{A}=\mathfrak{R}$. We also have the following extension of this result: $\mathfrak{A}$ is dense if and only if for any finite dimensional subspace $\subseteq$ of $\Re$ and any 1.t. $B$ in $\subseteq$ there exists an $A \in \mathfrak{A}$ that coincides with $B$ in $\mathfrak{S}$. This follows directly from the definition.

By using the well-ordering theorem we can prove the existence of a complement $\mathfrak{S}^{\prime}$ of any subspace $\mathfrak{S}$, that is, a subspace $\widetilde{S}^{\prime}$ such that $\Re=\widetilde{S}+\mathfrak{S}^{\prime}$, $\mathfrak{S} \wedge \mathfrak{S}^{\prime}=0\left(^{8}\right)$. Now if $\mathfrak{S}$ is finite-dimensional and $B$ is any l.t. in $\mathfrak{S}$ we can extend $B$ to an 1.t. $A$ in $\Re$ by defining $x A=x B$ for $x \in \mathcal{S}$ and $x^{\prime} B=0$ for $x^{\prime} \in \mathfrak{S}^{\prime}$. This proves that $\mathfrak{R}$ itself is dense. Moreover, it should be noted that $A$ is finite valued in the sense that the image space $\Re A$ is finite-dimensional. Hence we have also shown that the totality $\mathfrak{F}$ of finite valued 1.t. is a dense set.

To construct a third example of a dense set of 1.t. we assume that $\Re$ has a denumerable basis $u_{1}, u_{2}, \ldots$. Let $\mathfrak{U}_{n}$ be the totality of 1.t. that map $\Re_{n}=\left\{u_{1}, \cdots, u_{n}\right\}$ into itself and map $\Re_{n}^{\prime}=\left\{u_{n+1}, u_{n+2}, \cdots\right\}$ into 0 . Let $\mathfrak{U}$ be the join of all the $\mathfrak{U}_{n}$. Then if $x_{1}, \cdots, x_{k}$ and $y_{1}, \cdots, y_{k}$ are given and the $x$ 's are linearly independent, the $x$ 's and the $y$ 's are contained in a suitable $\Re_{N}$. Hence there is an $A$ in $\mathfrak{u}_{N}$ such that $x_{i} A=y_{i}$. This proves that $\mathfrak{U}$ is dense.

(3) See, for example, Jacobson [1, p. 18]. 
If $F_{1}$ and $F_{2} \in \mathscr{F}$ and $A \in R, \Re F_{1}+\Re F_{2}, \Re A F_{1}$ and $\Re F_{1} A$ are all finite dimensional. Thus $F_{1}+F_{2}, F_{1} A, A F_{1} \in \mathfrak{F}$ and so $\mathfrak{F}$ is a two-sided ideal in the ring $\mathfrak{R}$. It is evident that if $\Re$ is infinite-dimensional, $\mathfrak{F}<\mathbb{R}$ since $1 \notin \mathfrak{F}$. We shall now show that any dense subring of $\mathfrak{F}$ is simple. For this purpose we prove first the following lemma.

LEMMA 1. Let $\mathfrak{A}$ be an arbitrary dense ring of l.t. and let $\mathfrak{B}$ be a two-sided ideal in $\mathfrak{A}$ that contains a finite valued l.t. $B \neq 0$. Then if $\subseteq$ is any finite-dimensional subspace of $\Re$, there exists a projection $E \in \mathfrak{B}$ of $\Re$ on $\mathfrak{S}$.

Let $y_{1}, \cdots, y_{n}$ be a basis for $\Re B$ and let $x_{1}$ be a vector such that $x_{1} B=y_{1}$. We determine an 1.t. $A_{1} \in \mathfrak{A}$ such that $y_{1} A_{1}=x_{1}$ and $y_{i} A_{1}=0$ for $i \neq 1$. Then $B A_{1}=E_{1} \in \mathfrak{B}, \Re E_{1}=\left\{x_{1}\right\}$ and $x_{1} E_{1}=x_{1}$. Hence $E_{1}$ is a projection of $\Re$ on $\left\{x_{1}\right\}$. Now let $x$ be any vector not equal to 0 and let $C$ and $D$ be determined in $\mathfrak{A}$ so that $x C=x_{1}$ and $x_{1} D=x$. Then it can be verified that $E=C E_{1} D$ is a projection of $\mathfrak{R}$ on $\{x\}$. Since $E \in \mathscr{B}$ this proves the lemma for one-dimensional subspaces. We now assume it true for $k-1$-dimensional subspaces and let $\mathfrak{S}$ be $k$-dimensional. Let $\Im_{k-1}$ be a $k$-1-dimensional subspace of $\subseteq$ and let $E_{k-1} \in \mathfrak{B}$ be a projection of $\Re$ on $\mathfrak{S}_{k-1}$. We may decompose $\mathfrak{S}$ as $\mathfrak{S}_{k-1} \oplus \mathfrak{S}^{\prime}$ where $\mathfrak{S}^{\prime}$ is the one-dimensional subspace such that $\mathfrak{S}^{\prime} E_{k-1}=0$. Let $E^{\prime} \in \mathfrak{B}$ be a projection of $\Re$ on $\mathfrak{\Im}^{\prime}$ and set $E=E_{k-1}+E^{\prime}-E_{k-1} E^{\prime}$. Then if $x_{k-1} \in \Im_{k-1}$, $x_{k-1} E=x_{k-1}$, if $x^{\prime} \in \mathfrak{S}^{\prime}, x^{\prime} E=x^{\prime}$ and if $x$ is arbitrary, then $x E \in \mathfrak{S}$. Since $E \in \mathfrak{B}, E$ is the required projection.

We may now prove the following theorem.

TheOREM 1. Any dense ring $\mathfrak{A}$ of finite valued linear transformations in a vector space over a division ring is simple.

Let $\mathfrak{B}$ be a two-sided ideal not equal to 0 in $\mathfrak{A}$ and let $A$ be an arbitrary element of $\mathfrak{A}$. We determine a projection $E$ in $\mathfrak{B}$ of $\Re$ on $\Re A$. Then $A=A E \in \mathfrak{B}$ and so $B \in \mathfrak{A}$.

This proves in particular that $\mathfrak{F}$ and $\mathfrak{U}$ are simple rings. Concerning $\mathfrak{R}$ we have the following theorem.

Theorem 2. Any two-sided ideal $\mathfrak{B} \neq 0$ of $\mathfrak{R}$ contains $\mathfrak{F}$.

We note first that $\mathfrak{B} \wedge \mathfrak{F} \neq 0$. For otherwise $\mathfrak{B} \mathfrak{F}=\mathfrak{F} \mathfrak{B}=0$. Then if $B$ is any 1.t. in $\mathscr{B}$ and $u$ is a fixed vector not equal to 0 , any vector $x$ has the form $u F$ for some $F$ in $\mathfrak{F}$. Then $x B=u F B=0$. Hence $B=0$ so that $\mathscr{B}=0$ contrary to hypothesis. Thus we have proved that $\mathscr{B}$ contains finite valued 1.t. not equal to 0 . The proof of Theorem 1 then shows that $\mathfrak{B}$ contains $\mathfrak{F}$.

2. One-sided ideals in dense rings of linear transformations. If $\mathbb{S}$ is a subspace of $\Re$ we shall call a linear transformation an annihilator of $\mathfrak{S}$ if $\subseteq A=0$ and we shall call $A$ a retraction on $\subseteq$ if $\Re A \leqq \subseteq$. Now if $\mathfrak{A}$ is a subring of $\mathfrak{Q}$, it is clear that the totality of annihilators of $\subseteq$ contained in $\mathfrak{A}$ is a right ideal $\Im_{r}(\mathfrak{S})$. The totality of retractions on $\mathfrak{S}$ contained in $\mathfrak{A}$ is a left ideal 
$\Im_{l}\left(S_{)}\right.$. We note that $\Im_{l}\left(\Im_{)} \Im_{r}\left(S_{)}\right)=0\right.$ and if $\mathfrak{S}_{1} \leqq \Im_{2}$ then $\Im_{l}\left(\Im_{1}\right) \leqq \Im_{l}\left(\Im_{2}\right)$ and $\Im_{r}\left(\Im_{1}\right) \geqq \Im_{r}\left(\Im_{2}\right)$.

We suppose now that $\mathfrak{A}$ is dense and that $\subseteq$ is finite-dimensional. Then if $\subseteq \neq \Re, \Im_{r}(\subseteq) \neq 0$. For if $x_{1}, \cdots, x_{m}$ is a basis for $\subseteq$ and $x_{m+1}$ is a vector not in $\mathfrak{S}, x_{1}, \cdots, x_{m+1}$ are linearly independent. Hence there is an $A \in \mathfrak{A}$ such that $x_{1} A=\cdots=x_{m} A=0$ but $x_{m+1} A \neq 0$. Thus $A \neq 0$ and $A \in \Im_{r}(\Im)$. Our argument shows also that if $\mathfrak{S}_{1}$ and $\mathfrak{S}_{2}$ are finite-dimensional and $\mathfrak{S}_{2}>\mathfrak{S}_{1}$, then $\Im_{r}\left(\Im_{1}\right)>\Im_{r}\left(\Im_{2}\right)$. We note next that if $\left(\Im_{2}: \mathfrak{D}\right)=\left(\Im_{1}: \mathfrak{D}\right)+1$, then $\Im_{r}\left(\Im_{1}\right)$ is prime over $\Im_{r}\left(\mathfrak{S}_{2}\right)$ in the sense that there is no right ideal $\mathfrak{\Im}$ in $\mathfrak{A}$ such that $\Im_{r}\left(\Im_{1}\right)>\Im>\Im_{r}\left(\Im_{2}\right)$. For suppose that $\Im_{r}\left(\Im_{1}\right) \geqq \Im>\Im_{r}\left(\Im_{2}\right)$ and let $B \in \Im$, $B \notin \Im_{r}\left(\Im_{2}\right)$. Then if $x \in \Im_{2}, x \notin \Im_{1}, x B \neq 0$. Hence if $C$ is any l.t. in $\Im_{r}\left(\Im_{1}\right)$, there exists a $U$ in $\mathfrak{A}$ such that $(x B) U=x C$. Thus $C-B U$ annihilates $x$ and since $C-B U \in \Im_{r}\left(\Im_{1}\right), C-B U \in \Im_{r}\left(\Im_{2}\right)$. Since $B U \in \Im$ this proves that $C \in \Im$ and so $\Im=\Im_{r}\left(\Im_{1}\right)$.

Now $\Im_{r}(0)=\mathfrak{A}$. Hence we have shown in particular that $\Im_{r}(\{x\})$ is a maximal right ideal in $\mathfrak{A}$. If $\subseteq$ is $m$-dimensional, there is a chain of subspaces $0=\mathfrak{S}_{0}<\mathfrak{S}_{1}<\ldots<\mathfrak{S}_{m}=\mathfrak{S}$ such that $\left(\mathfrak{S}_{i}: \mathfrak{D}\right)=\left(\mathfrak{S}_{i-1}: \mathfrak{D}\right)+1$. Correspondingly we have a chain of right ideals $\mathfrak{A}=\Im_{0}>\Im_{1}>\cdots>\Im_{m}$ where $\Im_{j}=\Im_{r}\left(\Im_{j}\right)$ is prime over $\Im_{j+1}$. If $\Re$ itself is finite-dimensional, the sequence of right ideals leading to $\Im_{r}(\Re)$ is a composition series and it is well known that this implies that $\mathfrak{A}(=\mathfrak{R})$ satisfies the chain conditions for right ideals. On the other hand if $\Re$ is infinite-dimensional, we can find an infinite properly ascending sequence of finite-dimensional subspaces $\mathfrak{S}_{1}<\Im_{2}<\ldots$ in $\Re$. Then $\Im_{r}\left(\mathfrak{S}_{1}\right)>\Im_{r}\left(\mathfrak{S}_{2}\right)$ $>\cdots$. This proves the following theorem.

THEOREM 3. Let $\mathfrak{A}$ be a dense ring of linear transformations in $\mathfrak{R}$ over $\mathfrak{D}$. Then a necessary and sufficient condition that $\mathfrak{A}$ satisfy the descending chain condition for right ideals is that $\Re$ be finite-dimensional over $\mathfrak{D}$.

We have noted also that $\Im_{r}\{x\}$ is maximal if $x \neq 0$. It is evident that the intersection of all the ideals $\Im_{r}\{x\}$ is the 0 -ideal. Hence we have the following theorem.

THEOREM 4. If $\mathfrak{A}$ is a dense ring of linear transformations, $\mathfrak{A}$ contains maximal right ideals and the intersection of all the maximal right ideals in $\mathfrak{A}$ is the 0-ideal.

We assume next that $\mathfrak{A}$ is dense and that $\mathfrak{A}$ contains finite valued transformations not equal to 0 . Then if $\subseteq$ is any finite-dimensional subspace of $\Re$, by Lemma $1, \mathfrak{A}$ contains a projection $E$ of $\Re$ on $\mathfrak{S}$. If $\mathfrak{S}^{\prime}$ is the totality of vectors $y^{\prime}$ such that $y^{\prime} E=0$, we have $\Re=\subseteq+S^{\prime}, \subseteq \wedge \Im^{\prime}=0$. We consider now $\Im_{r}\left(\Im^{\prime}\right)$. By definition $E \in \Im_{r}\left(\Im^{\prime}\right)$. If $x=y+y^{\prime}$ where $y \in \Im, y^{\prime} \in \Im^{\prime}$ and $B \in \Im_{r}\left(\Im^{\prime}\right)$ then $x B=y B=x E B$. Hence $B=E B$ and so $\Im_{r}\left(\Im^{\prime}\right)=E \Re$. Suppose now that $\subseteq=\{u\}$ is one-dimensional and let $B \neq 0$ and $C$ be arbitrary elements of $\Im_{r}\left(\Im^{\prime}\right)$. Then $u B \neq 0$ and there is an 1.t. $U$ in $\mathfrak{A}$ such that 
$(u B) U=u C$. It follows that $C=B U$ and this implies that $\Im_{r}\left(\Im^{\prime}\right)$ is a minimal right ideal.

THEOREM 5. Let $\mathfrak{A}$ be a dense ring of linear transformations containing finite

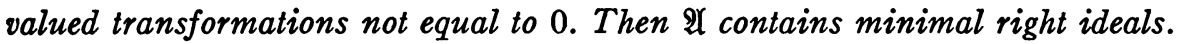

The theory of the left ideals $\Im_{l}(\mathfrak{S})$ is considerably less satisfactory than the foregoing. We note only the following result: If $\mathfrak{A}$ is dense and contains finite valued l.t. not equal to 0 and if $\subseteq$ is a finite-dimensional subspace of $\Re$, $\mathfrak{A}$ contains a projection $E$ of $\Re$ on $\mathfrak{S}$. Hence $\Im_{l}(\mathfrak{S})=\mathfrak{A} E \neq 0$. The join $\Re \Im_{l}(\mathfrak{S})$ of the spaces $\Re B$ where $B \in \Im_{l}(\mathfrak{S})$ is the whole space $\mathfrak{S}$. Hence if $\mathfrak{S}_{1}<\mathfrak{S}_{2}$ then $\Im_{l}\left(\mathfrak{S}_{1}\right)<\Im_{l}\left(\mathfrak{S}_{2}\right)$.

3. Irreducible rings of endomorphisms. We shall now shift our emphasis somewhat and begin with an arbitrary commutative group $\Re$ and with an irreducible ring $\mathfrak{A}$ of endomorphisms acting in $\mathfrak{R}$. Let $\mathfrak{D}$ be the centralizer of $\mathfrak{A}$ in the complete ring of endomorphisms in $\mathfrak{R}$. Thus $\mathfrak{D}$ consists of the endomorphisms $\alpha$ that commute with every $A$ in $\mathscr{A}$. We recall the well known lemma.

\section{LEMMA 2 (SCHUR). $\mathfrak{D}$ is a division ring $\left({ }^{4}\right)$.}

We now regard $\Re$ as a vector space over $\mathfrak{D}$ and we shall prove the following fundamental theorem.

THEOREM 6. Let $\mathfrak{A} \neq 0$ be an irreducible ring of endomorphisms acting in a commutative group $\Re$. Then if $\mathfrak{D}$ is the division ring of endomorphisms that commute with the elements of $\mathfrak{A}, \mathfrak{A}$ is dense in $\mathfrak{R}$ over $\mathfrak{D}$.

Proof. Let $x_{1}, \cdots, x_{k}$ be any $k \mathfrak{D}$-independent vectors and let $y_{1}, \cdots, y_{k}$ be arbitrary. We have to show that there is an $A$ in $\mathfrak{A}$ such that $x_{i} A=y_{i}$, $i=1, \cdots, k$. The case $k=1$ has been proved in $\S 1$. We assume now that $k=2$.

$k=2$. We prove first that there is an $A_{2}$ in $\mathscr{Q}$ such that $x_{1} A_{2}=0, x_{2} A_{2} \neq 0$. If this is not the case, then any equation of the form $x_{1} X=x_{1} Y$ for $X, Y \in \mathfrak{A}$ entails $x_{2} X=x_{2} Y$. Hence the mapping $y=x_{1} X \rightarrow x_{2} X=y^{\prime}$ is single-valued and since every $y$ is representable in the form $x_{1} X$, this mapping is defined in the whole of $\Re$. It is clear that

$$
\left(y_{1}+y_{2}\right)^{\prime}=y_{1}^{\prime}+y_{2}^{\prime}, \quad(y B)^{\prime}=y^{\prime} B,
$$

for all $B \in \mathfrak{A}$. Hence the mapping $y \rightarrow y^{\prime}$ is in $\mathfrak{D}$ and there is a $\gamma$ in $\mathfrak{D}$ such that $x_{2} X=\left(x_{1} X\right) \gamma$ for all $X$. Thus $\left(x_{2}-x_{1} \gamma\right) X=0$ and this implies that $x_{2}=x_{1} \gamma$, contrary to the linear independence of $x_{1}$ and $x_{2}$. This contradiction shows that there is an $A_{2}$ in $\mathfrak{A}$ such that $x_{1} A_{2}=0, x_{2} A_{2} \neq 0$. Similarly we have an $A_{1}$ such that $x_{1} A_{1} \neq 0, x_{2} A_{1}=0$. We now choose $B_{1}$ and $B_{2}$ so that $x_{1} A_{1} B_{1}=y_{1}$ and $x_{2} A_{2} B_{2}=y_{2}$. Then $A=A_{1} B_{1}+A_{2} B_{2}$ has the required properties.

(4) Jacobson [1, p. 57]. 
$k$ arbitrary. We assume the theorem for $k-1$ and as in the case $k=2$ it suffices to prove that there is an $A_{k}$ in $\mathfrak{A}$ such that $x_{1} A_{k}=\cdots=x_{k-1} A_{k}=0$, $x_{k} A_{k} \neq 0$. By the induction assumption there is a $B$ in $\mathscr{A}$ such that $x_{1} B=\cdots=x_{k-2} B=0$ but $x_{k} B \neq 0$. Let $\mathfrak{B}$ be the totality of mappings in $\mathfrak{A}$ that have this property. Assume first that $x_{k-1} B$ and $x_{k} B$ are $\mathfrak{D}$-independent. Then by the case $k=2$ there is a $C$ such that $x_{k-1} B C=0, x_{k} B C \neq 0$ and $A_{k}=B C$ is of the required type. Next let $x_{k-1} B$ and $x_{k} B$ be linearly dependent and we may assume that $x_{k-1} B=\left(x_{k} B\right) \beta$ where $\beta \neq 0$ is in $\mathfrak{D}$. We now choose a $D$ in $\mathscr{A}$ such that $x_{1} D=\cdots=x_{k-2} D=0$ but $\left(x_{k-1}-x_{k} \beta\right) D \neq 0$. If $x_{k} D=0$ and $x_{k-1} D \neq 0$ there is a $U$ in $\mathfrak{A}$ such that $x_{k-1} D U=x_{k-1} B$. Then for $A_{k}=B-D U$ we have $x_{1} A_{k}=\cdots=x_{k-1} A_{k}=0$ and $x_{k} A_{k}=x_{k} B \neq 0$. If $x_{k} D \neq 0$, $D \in \mathfrak{B}$. If either $x_{k-1} D=0$ or $x_{k-1} D$ and $x_{k} D$ are $\mathfrak{D}$-independent, then, as before, we may modify $D$ to obtain an $A_{k}$ of the required type. Hence we suppose that $x_{k-1} D=\left(x_{k} D\right) \delta$ where $\delta \neq 0$. Evidently $\delta \neq \beta$. Now there exists a $U$ in $\mathfrak{A}$ such that $x_{k-1} D U=x_{k-1} B$. Then $x_{k} D U=\left(x_{k-1} D\right) \delta^{-1} U=\left(x_{k-1} D U\right) \delta^{-1}$ $=\left(x_{k-1} B\right) \delta^{-1}=\left(x_{k} B\right) \beta \delta^{-1}$, that is, $x_{k}\left(B \beta \delta^{-1}-D U\right)=0$. Since $\beta \delta^{-1} \neq 1$, if we set $A_{k}=B-D U$ we have $x_{k} A_{k} \neq 0$, and so $A_{k}$ has the required properties.

As a first application of Theorem 6 we shall prove that if $\mathfrak{A}$ is a 2 -fold transitive ring of linear transformations in a vector space $\Re$ over $\mathfrak{D}$, then $\mathfrak{A}$ is dense. This will follow from the following theorem.

THEOREM 7. If $\mathfrak{A}$ is a 2-fold transitive ring of linear transformations in a vector space $\mathfrak{R}$ over $\mathfrak{D}$ then $\mathfrak{D}$ is the centralizer of $\mathfrak{A}$ in the complete ring of endomorphisms of $\Re$.

If $(\Re: \mathfrak{D})=1$ and $x \neq 0$ is in $\Re, \mathscr{R}$ consists of the mappings $y=x \xi \rightarrow(x \alpha) \xi=y^{\prime}$. $\mathfrak{A}$ is then a division ring anti-isomorphic to $\mathfrak{D}$ and the theorem is readily verified in this case $\left(^{(5)}\right.$. Now let $(\Re: D) \geqq 2$ and let $B$ be an endomorphism in $\Re$ that commutes with every $A$ in $\mathfrak{A}$. Then we assert that for every $x$ in $\Re, x$ and $x B$ are $\mathfrak{D}$-dependent. For otherwise there is an $A$ in $\mathfrak{A}$ such that $x A=0$ but $x B A \neq 0$ and this contradicts $A B=B A$. Hence if $x \neq 0, x B=x \beta_{x}, \beta_{x}$ in $\mathfrak{D}$. Now let $x$ and $y$ be linearly independent. Then $x B=x \beta_{x}$ and $y B=y \beta_{y}$. There is an $A$ in $\mathfrak{A}$ such that $x A=y$. Since $A B=B A$ this implies that $\beta_{x}=\beta_{y}=\beta$. Since $x \alpha$ and $y$ are linearly independent we have $(x \alpha) B=(x \alpha) \beta$ and this shows that $B=\beta \in \mathfrak{D}$.

As an immediate consequence of Theorems 6 and 7 we have the following theorem.

THEOREM 8. If $\mathfrak{A}$ is a 2-fold transitive ring of linear transformations in $\Re$ over $\mathfrak{D}, \mathfrak{A}$ is a dense ring.

4. Structure of simple rings that contain minimal right ideals. Let $\mathfrak{A}$ be an abstract simple ring that contains a minimal right ideal $\Im$. We assume

(5) Jacobson [1, p. 22]. 
also that $\mathfrak{A}$ is not a zero ring. Hence $\mathfrak{U}^{2}=\mathfrak{A} \neq 0$. It follows that $\mathfrak{\Im} \mathfrak{A} \neq 0$. For otherwise $\mathfrak{B}=\mathfrak{A} \mathfrak{Y}+\mathfrak{Y}$ is a nilpotent two-sided ideal and $\mathfrak{B}=\mathfrak{A}$ contrary to $\mathfrak{A}^{2} \neq 0$. It is known that $\Im=e \mathfrak{A}$ where $e$ is an idempotent element $\left(^{(}\right)$.

Each element $a$ of $\mathfrak{A}$ defines an endomorphism $\bar{a}$ in $\Im$ by the equation $x \bar{a}=x a, x \in \Im$. The set $\overline{\mathfrak{A}}$ of these endomorphisms is a subring not equal to 0 of the ring of endomorphisms of $\Im$ and the correspondence $a \rightarrow \bar{a}$ is a homomorphism. Since $\mathfrak{A}$ is simple, $a \rightarrow \bar{a}$ is an isomorphism. Since $\Im$ is minimal, $\overline{\mathscr{P}}$ is an irreducible ring of endomorphisms. Hence if $\mathfrak{D}$ denotes the division ring consisting of the endomorphisms $\alpha, \beta, \ldots$ that commute with all the $\bar{a}$, $\overline{\mathfrak{A}}$ is a dense ring of linear transformations in $\Im$ regarded as a vector space over $\mathfrak{D}$.

We shall show next that $\overline{\mathfrak{A}}$ consists of finite valued 1.t. We prove first that $(\Im \bar{e}: \mathfrak{D})=1$. For otherwise we should have two $\mathfrak{D}$-independent elements $x, y$ in $\Im$ such that $x \bar{e}=x, y \bar{e}=y$. Then there is a $\bar{d} \in \overline{\mathscr{A}}$ such that $x \bar{d}=0$ and $y \bar{d} \neq 0$ and if $\bar{b}=\bar{e} \bar{d}, x \bar{b}=0, y \bar{b} \neq 0$. Since $\bar{b} \in \bar{e} \overline{\mathfrak{A}}=\bar{\Im}$, this shows that $\bar{\Im}_{\mathcal{A}} \wedge \bar{\Im}_{r}\{x\}$ $\neq 0$, where $\bar{\Im}_{r}\{x\}$ denotes as usual the annihilator of the subspace $\{x\}$. Since $\bar{\Im}$ is minimal, $\bar{\Im} \wedge \bar{\Im}_{r}\{x\}=\bar{\Im}$ and this contradicts the equation $x \bar{e}=x$. We have therefore proved our assertion that $(\Im \bar{e}: \mathfrak{D})=1$. Thus $\bar{e}$ is finite valued. Now the subset of finite valued transformations belonging to $\overline{\mathfrak{A}}$ is a two-sided ideal. Since we have shown that this subset is not equal to 0 it follows that $\overline{\mathscr{A}}$ contains only finite valued l.t. This together with Theorems 1 and 5 proves the following structure theorem.

THEOREM 9. If $\mathfrak{A}$ is a simple ring (not a zero ring) that contains a minimal right ideal, then $\mathfrak{A}$ is isomorphic to a dense ring of finite valued linear transformations in a vector space over a division ring. Conversely, any dense ring of finite valued linear transformations in a vector space over a division ring is simple and contains minimal right ideals.

As a corollary of this theorem and of Theorem 3 we have the fundamental Wedderburn-Artin structure theorem.

THEOREM 10. If $\mathfrak{A}$ is a simple ring (not a zero ring) that satisfies the descending chain condition for right ideals, then $\mathfrak{A}$ is isomorphic to the complete ring of linear transformations in a suitable finite-dimensional vector space over a division ring, and conversely.

Another consequence of the main theorem is a result noted previously by Artin and Whaples, namely, if $\mathfrak{A}$ is simple and contains a minimal right ideal, then $\mathfrak{A}$ satisfies the descending chain condition for r $r^{\prime}$ ht ideals if and only if $\mathfrak{A}$ has an identity $\left({ }^{7}\right)$. This is clear since the identity 1 of $\mathfrak{A}$ is finite valued if and only if the space is finite dimensional. Other simple consequences of the

(6) Jacobson [1, p. 64].

(7) Artin and Whaples [1, p. 91]. 
main theorem and of the discussion of $\$ 2$ are that if $\mathfrak{A}$ is simple and contains a minimal right ideal, then $\mathfrak{A}$ contains maximal right ideals and the intersection of all the maximal right ideals of $\mathfrak{A}$ is the 0 -ideal.

We consider now the question of the uniqueness of the representation of $\mathfrak{A}$ as a dense ring of linear transformations. We suppose that $\Re$ is an arbitrary commutative group, that $\overline{\mathfrak{A}}$ is an irreducible subring not equal to 0 of the ring of endomorphisms of $\Re$ and that $\overline{\mathfrak{A}}$ is a homomorphic image of $\mathfrak{A}$. Let $a \rightarrow \bar{a}$ be the correspondence between $\mathfrak{A}$ and $\overline{\mathfrak{A}}$. Since $\mathfrak{A}$ is simple and $\overline{\mathfrak{A}} \neq 0$ our correspondence is in reality an isomorphism. Let $\Im$ be a minimal right ideal in $\Re$. Then there is an $x$ in $\Re$ such that $x \bar{\Im} \neq 0$. Since the set $x \bar{\Im}$ is a subgroup invariant under $\mathfrak{A}, x \bar{\Im}=\Re$ and since $\Im$ is minimal, $x \bar{b}=0$ for $b$ in $\Im$ only if $b=0$. Thus the correspondence $b \rightarrow x b \equiv b^{\prime}$ is an isomorphism between $\Im$ and $\Re$. Since $\overline{b a}=\bar{b} \bar{a},(b a)^{\prime}=b^{\prime} \bar{a}$ and so $b \rightarrow b^{\prime}$ is an operator isomorphism. It follows from this that if $\mathfrak{D}$ and $\overline{\mathfrak{D}}$, respectively, are the centralizers of the multiplications $b \rightarrow b a$ in $\Im$ and of the ring $\overline{\mathscr{A}}$ in $\Re$, then there is an isomorphism $\alpha \rightarrow \bar{\alpha}$ between $\mathfrak{D}$ and $\bar{D}$ such that $(b \alpha)^{\prime}=b^{\prime} \bar{\alpha}$ for all $b$. By Theorem 6 , we know that $\overline{\mathfrak{A}}$ is dense in $\Re$ over $\mathfrak{D}$. Moreover, by our isomorphisms, and the fact that the multiplications in $\Im$ are all finite valued, we see that the l.t. of $\overline{\mathfrak{A}}$ are all finite valued.

Now suppose that $\Re_{1}$ and $\Re_{2}$ are vector spaces over $\mathfrak{D}_{1}$ and $\mathfrak{D}_{2}$, respectively, and that $\mathfrak{A}_{1}$ and $\mathfrak{A}_{2}$ are isomorphic dense rings of finite valued transformations in these spaces. Let $a_{1} \rightarrow a_{2}$ be a definite isomorphism between these rings. We may regard $\mathfrak{A}_{1}$ and $\mathfrak{A}_{2}$ as isomorphic images of the same simple ring $\mathfrak{A}$ that contains a minimal right ideal $\mathfrak{Y}$. Let the isomorphism between $\mathfrak{A}$ and $\mathfrak{A}_{i}, i=1,2$, be $a \rightarrow a_{i}$. By Theorem $6, \mathfrak{D}_{i}$ is the centralizer of $\mathfrak{A}_{i}$. Hence by the above argument there is an isomorphism $b \rightarrow b_{i}$ between $\Im$ and $\Re_{i}$ and an isomorphism $\alpha \rightarrow \alpha_{i}$ between $\mathfrak{D}$, the centralizer of the multiplications in $\Im$, and $\mathfrak{D}_{i}$ such that $b \alpha \rightarrow b_{i} \alpha_{i}$ and $b a \rightarrow b_{i} a_{i}$. It follows that the correspondences $b_{1} \rightarrow b_{2}, b_{i} \in \Re_{i}, \alpha_{1} \rightarrow \alpha_{2}, \alpha_{i} \in \mathfrak{D}_{i}$, are isomorphisms and that $b_{1} \alpha_{1} \rightarrow b_{2} \alpha_{2}$ and $b_{1} a_{1} \rightarrow b_{2} a_{2}$. This proves the following important uniqueness theorem.

TheoRem 11. Let $\Re_{1}$ and $\Re_{2}$ be vector spaces over $\mathfrak{D}_{1}$ and $\mathfrak{D}_{2}$, respectively, and let $\mathfrak{A}_{1}$ and $\mathfrak{A}_{2}$ be isomorphic dense rings of finite valued linear transformations acting in these spaces. Then if $a_{1} \rightarrow a_{2}$ is a given isomorphism between $\mathfrak{A}_{1}$ and $\mathfrak{A}_{2}$, there exists an isomorphism $x_{1} \rightarrow x_{2}$ between $\Re_{1}$ and $\Re_{2}$ and an isomorphism $\alpha_{1} \rightarrow \alpha_{2}$ between $\mathfrak{D}_{1}$ and $\mathfrak{D}_{2}$ such that $x_{1} \alpha_{1} \rightarrow x_{2} \alpha_{2}$ and $x_{1} a_{1} \rightarrow x_{2} a_{2}$.

Theorem 11 shows that two dense rings of finite valued 1.t. are not isomorphic if the vector spaces in which they act are not isomorphic. If the spaces are isomorphic, we may without loss of generality identify them. Then our theorem assumes the following form:

THEOREM 12. Let $\mathfrak{R}$ be a vector space over a division ring $\mathfrak{D}$ and let $\mathfrak{A}_{1}$ and $\mathfrak{A}_{2}$ be two isomorphic dense rings of finite valued linear transformations in $\Re$ over $\mathfrak{D}$. 
Then if $a_{1} \rightarrow a_{2}$ is a given isomorphism between $\mathfrak{A}_{1}$ and $\mathfrak{A}_{2}$, there exists a semilinear transformation $\mathfrak{S}$ in $\Re$ over $\mathfrak{D}$ such that $a_{2}=S^{-1} a_{1} S$ for all $a_{1}\left({ }^{8}\right)$.

If $\mathfrak{A}$ is a simple, nonzero ring that contains a minimal left ideal, it is known that $\mathfrak{A}$ contains a minimal right ideal $\left({ }^{9}\right)$. Our discussion is therefore applicable to these rings also.

5. Simple rings that contain maximal right ideals. We shall suppose now that $\mathfrak{A}$ is a simple ring that contains a maximal right ideal $\mathfrak{Y}$. By the well ordering theorem we may prove that any ring with an identity has this property. As before, we consider the right multiplications $x \rightarrow x a$ for $x$ in $\mathfrak{A}$ and $a$ in $\mathfrak{A}$. These mappings are endomorphisms that induce endemorphisms in $\Im$ and also in the difference group $\Re=\mathfrak{A}-\mathfrak{\Im}$. We denote the endomorphism induced by $x \rightarrow x a$ in $\Re$ by $\bar{a}$ and we denote the set of $\bar{a}$ by $\overline{\mathfrak{A}}$. Since $\Im$ is maximal, $\overline{\mathfrak{A}}$ is an irreducible set of endomorphisms. If $\overline{\mathfrak{A}}=0, \mathfrak{U}^{2} \leqq \mathfrak{Y}$, and this implies that $\mathfrak{A}$ is a zero-ring. We exclude this case from further consideration. Then $\overline{\mathfrak{A}}$ is a ring not equal to 0 and the correspondence $a \rightarrow \bar{a}$ is an isomorphism between $\mathfrak{A}$ and $\overline{\mathfrak{A}}$.

Now let $\mathfrak{D}$ be the centralizer of $\overline{\mathfrak{A}}$ in the ring of endomorphisms of $\Re$. Then by Theorem $6, \overline{\mathfrak{A}}$ is a dense ring of linear transformations in $\Re$ over $\mathfrak{D}$. Since $\mathfrak{A}$ is simple, we have the following two possibilities: either $\overline{\mathfrak{A}}$ consists exclusively of finite valued l.t. or every l.t. not equal 0 in $\overrightarrow{\mathfrak{A}}$ is infinite valued. We have seen that the former case obtains if and only if $\mathfrak{A}$ has a minimal right ideal. This proves the following theorem.

THEOREM 13. Let $\mathfrak{A}$ be a simple ring (not a zero ring) that contains a maximal right ideal. Then $\mathfrak{A}$ is isomorphic to a dense ring $\overrightarrow{\mathfrak{A}}$ of linear transformations in a suitable vector space over a division ring. If $\mathfrak{A}$ contains a minimal right ideal, $\overline{\mathfrak{A}}$ consists exclusively of finite valued linear transformations, otherwise every linear transformation not equal to 0 in $\overline{\mathfrak{A}}$ is infinite valued.

If $\mathfrak{A}$ has an identity, the division ring $\mathfrak{D}$ obtained from the maximal right ideal $\Im$ may be obtained in the following manner. We have $\Re=[1] \Re$ where [1] denotes the coset $1+\Im$. Let $\beta \in \mathfrak{D}$ and suppose that $[1] \beta=[b]=b+\Im$. Then if $[x]$ is any coset, we have $[x]=[1] x$ and $[x] \beta=([1] x) \beta=([1] \beta) x$ $=[b] x=[b x]$. Since $[0] \beta=[0]$, the elements $b x \in \Im$ for every $x$ in $\Im$. Now we shall define the normalizer $\left.{ }^{10}\right) \mathscr{B}$ of the right ideal $\Im$ to be the totality of elements $b$ such that $b \mathfrak{Y} \leqq \mathfrak{F}$ is a subring of $\mathscr{A}$ containing $\mathfrak{Y}$ and is in fact the largest subring of $\mathfrak{A}$ in which $\mathfrak{I}$ is a two-sided ideal. Our computation above shows that the elements of $\mathfrak{D}$ are of the form $[x] \rightarrow[b x]$ where $b \in \mathfrak{B}$. Conversely, it is readily seen that if $b$ is any element of $\mathfrak{B}$ the mappings of

(8) A transformation $S$ in a vector space is called semi-linear if it is an endomorphism and $(x \alpha) S=(x S) \bar{a}$ where $\alpha \rightarrow \bar{a}$ is an automorphism in $\mathfrak{D}$.

( ${ }^{\circ}$ Artin and Whaples $[1$, p. 92].

$\left({ }^{10}\right)$ This is the "Eigenring" of an ideal first defined by Ore [1]. Cf. also Fitting [1]. 
this form are single valued and belong to $\mathfrak{D}$. Two elements $b_{1}$ and $b_{2}$ give rise to the same mapping $\beta \in \mathfrak{D}$ if and only if $b_{1}-b_{2} \in \Im$. It follows from this that $\mathfrak{D}$ is anti-isomorphic to the difference ring $\mathscr{B}-\mathfrak{Y}$.

We shall give now an example of a simple ring that contains no minimal right ideals.

Example. Let $\Phi$ be a differential field of characteristic 0 such that the field $\Phi_{0}$ of constants is a proper subfield of $\Phi$. Let $\mathfrak{A}$ be the domain of differential polynomials $D^{m} a_{1}+D^{m-1} a_{1}+\cdots+a_{m}$ where the $a_{i} \in \Phi$. We have the commutation rule $a D=D a+a^{\prime}$ where $a^{\prime}$ is the derivative of $a$. Then it is known that every right ideal and every left ideal in $\mathfrak{A}$ is principal. Suppose now that $\Im^{*}$ is a two-sided ideal not equal to 0 . Then it is easily shown that $\mathfrak{S}^{*}=\mathfrak{A} A^{*}=A^{*} \mathfrak{A}$ where $A^{*}=D^{m} a_{0}+D^{m-1} a_{1}+\cdots+a_{m}, a_{0} \neq 0\left({ }^{11}\right)$. Thus if $B$ is any element of $A, B A^{*}$ is divisible on the left by $A^{*}$, say $B A^{*}=A^{*} \bar{B}$. In particular, for $B=b \in \Phi$, considerations of degree show that $b \in \Phi$. A comparison of leading coefficients shows that $b=b$ and so $A^{*}$ commutes with all the elements of $\Phi$. Now $b D^{k}=D^{k} b+C_{k, 1} D^{k-1} b^{\prime}+\cdots+b^{(k)}$. Hence $0=b A^{*}-A^{*} b$ $=C_{m, 1} D^{m-1} b^{\prime} a_{0}+\cdots$. If $m>0$ this implies that $b^{\prime}=0$ for all $b$ in $\Phi$ contrary to assumption. Thus $A^{*}=a \in \Phi$ and $\mathfrak{\Im}^{*}=\mathfrak{A}$. This proves the simplicity of $\mathfrak{A}$. If $\Im=A \mathfrak{A}$ is any right ideal not equal to 0 and $B$ is an element of positive degree in $\mathfrak{A}, \Im>A B \mathfrak{A}>0$. This shows that $\mathfrak{A}$ contains no minimal right ideals. It is not difficult to construct maximal ideals $\Im_{1}$ and $\Im_{2}$ in $\mathfrak{A}$ such that if $\mathfrak{B}_{1}$ and $\mathfrak{B}_{2}$ are the corresponding normalizers, then $\mathfrak{B}_{1}-\Im_{1}$ and $\mathfrak{B}_{2}-\Im_{2}$ are not isomorphic $\left.{ }^{12}\right)$. Thus no uniqueness theorem of the form of Theorem 11 is valid in this case.

\section{NoN-ASSOCIATIVE SIMPLE RINGS}

6. Multiplication ring of a non-associative simple ring. If $\mathfrak{A}$ is an arbitrary non-associative ring $\left({ }^{13}\right)$ we define the right multiplication $a_{r}$ as the mapping $x \rightarrow x a, x$ in $\mathfrak{A}$, and the left multiplication $a_{l}$ as $x \rightarrow a x$. These are endomorphisms in the additive group of $\mathfrak{A}$ and they generate a subring $\mathfrak{M}$ of the complete ring of endomorphisms of $\mathfrak{A}$. We call $\mathfrak{M}$ the multiplication ring of $\mathfrak{A}$. A subgroup $\mathfrak{B}$ of $\mathfrak{A}$ is a two-sided ideal if $\mathfrak{B}$ is invariant under all the right and left multiplications, or, what is equivalent, if $\mathscr{B}$ is invariant under the endomorphisms belonging to $\mathfrak{M}$. Thus $\mathfrak{A}$ is simple if and only if $\mathfrak{M}$ is an irreducible ring of endomorphisms.

We shall call the centralizer $\mathfrak{C}$ of $\mathfrak{M}$ in the ring of endomorphisms of $\mathfrak{A}$ the multiplication centralizer of $\mathfrak{A}$. The elements of $\mathfrak{E}$ are the endomorphisms $\gamma$ that satisfy

$$
(x y) \gamma=(x \gamma) y=x(y \gamma) .
$$

(11) Jacobson [1, p. 37].

(12) This is indicated in Jacobson $[3$, p. 506].

$\left({ }^{13}\right)$ By a non-associative ring we, of course, mean a ring which is not necessarily associative. 
Hence, if $\gamma, \delta \in \mathfrak{C}$

$$
\begin{aligned}
(x y) \gamma \delta & =((x \gamma) y) \delta=(x \gamma)(y \delta) \\
& =(x(y \gamma)) \delta=(x \delta)(y \gamma)
\end{aligned}
$$

and this proves that $(x y) \gamma \delta=(x y) \delta \gamma$. Thus if $\mathfrak{Q}^{2}$ is the subring consisting of the finite sums of products $a b$, and $\mathfrak{A}^{2}=\mathfrak{A}$, then $\gamma \delta=\delta \gamma$. This shows that in this case $\mathbb{E}$ is a commutative ring.

Now let $\mathfrak{A}$ be a non-associative simple ring that is not a zero ring. Then $\mathfrak{M}$ is an irreducible ring of endomorphisms. Hence $\mathbb{C}$ is a division ring. Since $\mathfrak{A}^{2}$ is a two-sided ideal not equal to $0, \mathfrak{A}^{2}=\mathfrak{A}$. Hence $\mathfrak{E}$ is a field. By Theorem 6 , $\mathfrak{A}$ is a dense ring of linear transformations in $\mathfrak{A}$ over $\mathfrak{C}$. This proves the following theorem.

THEOREM 14. Let $\mathfrak{A}$ be a non-associative ring that is simple and not a zero ring. Then the multiplication centralizer $\mathfrak{S}$ of $\mathfrak{A}$ is a field and the multiplication ring $\mathfrak{M}$ is a dense ring of linear transformations in $\mathfrak{A}$ over $\mathbb{S}\left({ }^{14}\right)$.

By equation (1), $\mathfrak{A}$ may be regarded as an algebra over $\mathfrak{E}$. Obviously $\mathfrak{A}$ is a simple algebra.

A similar discussion applies to non-associative algebras. If $\Phi$ is the underlying field, then $\Phi \leqq \mathfrak{C}$. Now by $(1),(a \gamma)_{r}=a_{r} \gamma$ and $(a \gamma)_{l}=a_{l} \gamma$. Hence $\mathfrak{M E}=\mathbb{E} \mathfrak{M} \leqq \mathfrak{M}$ and in particular $\mathfrak{M}$ is closed under multiplication by the elements of $\Phi$. It follows that if $x$ is any element of $\mathfrak{R}$, the totality $x \mathfrak{M}$ of elements $x M, M$ in $\mathfrak{M}$, is a subspace over $\Phi$ as well as an ideal in $\mathfrak{A}$. If $\mathfrak{A}$ is a simple algebra that is not a zero algebra, we can show that if $x \mathfrak{M}=0$ then $x=0$. Hence $x \mathfrak{M}=\mathfrak{A}$ for every $x \neq 0$ and this proves that $\mathfrak{A}$ is a simple ring. The converse is obvious. Theorem 14 may now be stated for algebras also.

We suppose now that $(\mathfrak{A}: \Phi)=n<\infty$. In this case $\mathfrak{M}$ and $\mathfrak{E}$ are subalgebras of the complete algebra of linear transformations in $\mathfrak{A}$ regarded as a space over $\Phi$. Hence $(\mathfrak{M}: \Phi)$ and $(\mathfrak{C}: \Phi) \leqq n^{2}$. If $(\mathfrak{C}: \Phi)=r$ we know that $n=m r$ and $(\mathfrak{A}: \mathfrak{C})=m$. By Theorem $14, \mathfrak{M}$ is the complete set of linear transformations in $\mathfrak{A}$ over $\mathfrak{C}$. Thus $(\mathfrak{M}: \mathfrak{C})=m^{2}$ and $(\mathfrak{M}: \Phi)=m^{2} r$. This proves the following known $\left({ }^{15}\right)$ theorem.

THEOREM 15: Let $A$ be a simple, nonzero, non-associative algebra with a finite basis over a field $\Phi$ and let $(\mathfrak{A}: \Phi)=n$. Then if $\mathbb{S}$ is the multiplication centralizer, $\mathbb{S}$ is a finite extension field over $\Phi$ and (א: $\Phi)$ is a divisor of $n$. The multiplication algebra $\mathfrak{M}$ is the complete set of linear transformations of $\mathfrak{A}$ over $\mathfrak{E}$.

It is clear from this theorem that in the finite basis case, $\mathbb{C}$ is a subset of $\mathfrak{M}$. Hence $\mathfrak{C}$ is the center of $\mathfrak{M}$.

7. The center of a non-associative simple ring. We shall define the center $\mathfrak{S}_{0}$ of a non-associative ring $\mathfrak{A}$ to be the totality of elements $c$ that commute

(14) This theorem for associative algebras is due to Artin and Whaples [1, p. 98].

15) Jacobson [2]. 
with every element of $\mathfrak{A}$ and that associate with every pair of elements $x, y$ in $\mathfrak{A}$ in the sense that

$$
(x y) c=x(y c), \quad(x c) y=x(c y), \quad(c x) y=c(x y) .
$$

We note that the elements of $\mathfrak{E}_{0}$ may also be characterized by the equation $x c=c x$ and

$$
(x y) c=x(y c)=(x c) y .
$$

For by (2) we have $(x y) c=x(y c)$ and $(x c) y=x(c y)=x(y c)$ since $y c=c y$. Hence (3) holds. Conversely, if (3) holds and $c$ commutes with every $x$, then $(x y) c=c(x y)=x(y c)=x(c y)=(x c) y=(c x) y$. Hence (2) is satisfied and in fact all the products in (2) are equal. Thus the conditions that $c \in \mathfrak{E}_{0}$ are that $c_{r}=c_{l}$ and that this endomorphism belongs to the multiplication centralizer $\mathfrak{E}$. If $c_{1}, c_{2} \in \mathfrak{G}_{0}$ it is clear that $c_{1} \pm c_{2} \in \mathfrak{G}_{0}$ and $\left(c_{1} \pm c_{2}\right)_{r}=c_{1 r} \pm c_{2 r}$. Also $\left(x c_{1}\right) c_{2}=x\left(c_{1} c_{2}\right)$ so that $\left(c_{1} c_{2}\right)_{r}=c_{1 r} c_{2 r}$ and similarly $\left(c_{1} c_{2}\right)_{l}=\left(c_{2} c_{1}\right)_{l}=c_{12} c_{2 l}=c_{1 r} c_{2 r}=\left(c_{1} c_{2}\right)_{r}$. Evidently $c_{1 r} c_{2 r} \in \mathfrak{C}$. Hence we have proved that $c_{1} c_{2} \in \mathfrak{E}_{0}$. Now let $\gamma$ be an arbitrary element of $\mathfrak{E}$. Then if $c \in \mathfrak{E}_{0}, x(c \gamma)=(x \gamma) c=(x c) \gamma=(c x) \gamma=(c \gamma) x$ and so $(c \gamma)_{r}=(c \gamma)_{l}=c_{r} \gamma$. This proves that $c \gamma \in \mathfrak{G}_{0}$. Thus $\mathfrak{S}_{0}$ is a commutative and associative subring of $\mathfrak{A}$ and $\mathfrak{E}_{0}$ is invariant under the endomorphisms $\gamma \in \mathfrak{C}$. We have also shown that the correspondence $c \rightarrow c_{r}$ is a homomorphism that commutes with all $\gamma$ in $\mathbb{E}$ between $\mathfrak{S}_{0}$ and a subring $\mathfrak{E}_{0 r}$ of $\mathfrak{E}$.

We suppose now that $\mathfrak{A}$ is simple and not a zero ring. Then we note first that the correspondence $\dot{c} \rightarrow c_{r}$ is an isomorphism. For if $c_{r}=0$ then $\{c\}$, the additive group generated by $c$, is a two-sided ideal in $\mathfrak{A}$. If $\mathfrak{A}=\{c\}, \mathfrak{A}$ is a zero ring. Hence $\{c\}=0$ and $c=0$. We remark next that $\mathfrak{E}_{0 r}$ is an ideal in (E since $c_{r} \gamma=(c \gamma)_{r}$. Since, by Theorem 14 , $\mathfrak{C}$ is a field, either $\mathfrak{E}_{0 r}=0$ or $\mathfrak{E}_{0 r}=\mathfrak{C}$. In the former case $\mathfrak{E}_{0}=0$. In the latter case $\mathfrak{S}_{0}$ is a field and if 1 is the identity of $\mathfrak{S}_{0}$, then $1_{r}=1_{l}$ is the identity transformation. Thus 1 is the identity of $\mathfrak{A}$ also. We have therefore proved the following theorem.

THEOREM 16. If $\mathfrak{A}$ is a simple, nonzero, non-associative ring, then a necessary and sufficient condition that $\mathfrak{A}$ have an identity is that its center $\mathfrak{S}_{0} \neq 0$. If the condition is satisfied, $\mathfrak{S}_{0}$ is a field and the multiplication centralizer consists of the multiplications by the elements of $\mathfrak{S}_{0}$.

These results are valid for algebras also since, as we have seen, a nonzero algebra $\mathfrak{A}$ is simple if and only if $\mathfrak{A}$ is a simple ring.

8. Extension of the field of a vector space. One of the most powerful methods of studying a non-associative algebra $\mathfrak{A}$ of a finite dimensionality is to consider the extension algebras $\mathfrak{A}_{\Sigma}, \Sigma$ a field containing the underlying field $\Phi$. It is often possible to determine the structure of a certain extension $\mathfrak{A}_{\Sigma}$ and then to use this information to obtain the structure of $\mathfrak{A}$. This method has been particularly successful in the study of simple Lie algebras. In the 
next section we shall lay the foundations for a theory of extensions of simple algebras of infinite dimensionality. As a necessary preliminary we consider here the definition of an extension $\Re_{\Sigma}$ of a vector space $\Re$ over $\Phi$ by means of the extension field $\Sigma$ of $\Phi$.

Let $\Re$ be a vector space over a field $\Phi$ and let $\Sigma$ be an abstract field that contains a subfield isomorphic to $\Phi$. Now we wish to construct a vector space $\Re_{\Sigma}$ over a field (isomorphic to) $\Sigma$ with the following properties:

1. $\Re_{\Sigma}$ regarded as a space over $\Phi$ contains a subspace (isomorphic to) $\Re$.

2. Any elements $x_{1}, \cdots, x_{n}$ of $\Re$ that are $\Phi$-independent are also $\Sigma$-independent.

3. The smallest subspace over $\Sigma$ of $\Re_{\Sigma}$ that contains $\Re$ is $\Re_{\Sigma}$ itself.

The definition of an extension $\Re_{\Sigma}$ by means of a basis is well known and in a recent paper Artin and Whaples( $\left.{ }^{16}\right)$ have given a definition without using bases. We follow their method here. We consider first the totality $\mathfrak{U}$ of forms $x_{1} \sigma_{1}+x_{2} \sigma_{2}+\cdots+x_{m} \sigma_{m}$ of arbitrary length $m$ in which the $x_{i} \in \Re$ and the $\sigma_{i} \in \Sigma$. The order of the terms in $\sum x_{i} \sigma_{i}$ is immaterial and we define the sum of two forms $\sum_{1}^{m} x_{i} \sigma_{i}+\sum_{m+1}^{n} x_{j} \sigma_{j}$ as $\sum_{1}^{n} x_{k} \sigma_{k}$. Relative to this definition $\mathfrak{u}$ is a commutative semi-group. Now we call $\sum x_{i} \sigma_{i}$ a null-form if there exists a substitution $x_{i}=\sum y_{k} \alpha_{k i}, \alpha_{k i}$ in $\Phi$, so that the resulting form $\sum y_{k}\left(\alpha_{k i} \sigma_{i}\right)$ has 0 coefficients, that is, $\sum \alpha_{k_{i}} \sigma_{i}=0$. It can be shown that $\sum x_{i} \sigma_{i}$ is a null form if and only if there exists a substitution $\sigma_{i}=\sum \beta_{i k} \tau_{k}, \beta_{i k}$ in $\Phi$, so that the coefficients $\sum x_{i} \beta_{i k}$ of the $\sigma_{\dot{k}}$ in the form $\sum x_{i} \beta_{i k} \sigma_{k}$ are all 0 . If the $x$ 's are linearly independent in $\Re$ and not all the $\sigma$ 's are 0 or if the $\sigma$ 's are linearly independent in $\Sigma$ (over $\Phi$ ) and not all the $x$ 's are 0 then $\sum x_{i} \sigma_{i}$ is not a null-form.

It is readily seen that the totality $\mathfrak{R}$ of null-forms is a subsemi-group of $\mathfrak{U}$. Now we define $-x$ for $x=\sum x_{i} \sigma_{i}$ to be the form $\sum x_{i}\left(-\sigma_{i}\right)$ and we define $x \equiv y(\bmod \mathfrak{R})$ if $x+(-y) \in \mathfrak{N}$. It can be shown that this notion of congruence is an equivalence relation and that congruences can be added. Hence if we let $\Re_{\Sigma}$ denote the set of congruence classès, $\Re_{\Sigma}$ is also a commutative semigroup. Moreover, it can be seen that $\Re_{\Sigma}$ is a group with $\mathfrak{R}$ as its zero and the class of $-x$ as the negative of the class of $x$.

We shall denote the elements of $\Re_{\Sigma}$ by their representatives $\sum x_{i} \sigma_{i}$ in $\mathfrak{u}$. When this is done we have the following criterion: $\sum x_{i} \sigma_{i}=\sum y_{i} \tau_{j}$ if and only if when the $x$ 's and the $y$ 's are expressed in terms of the same set of linearly independent vectors $u_{1}, \cdots, u_{r}$, the resulting coefficient of each $u_{k}$ on the left-hand side is the same as that on the right-hand side. In $\Re_{\Sigma}$ we have $\sum_{1}^{m} x_{i} \sigma_{i}+\sum_{m+1}^{n} x_{j} \sigma_{j}=\sum_{1}^{n} x_{k} \sigma_{k}$.

It is now easy to verify that for each $\sigma \in \Sigma$, the mapping $x=\sum x_{i} \sigma_{i}$ $\rightarrow \sum x_{i}\left(\sigma_{i} \sigma\right)$ is single valued and an endomorphism in $\Re_{\Sigma}$. The totality of these endomorphisms is a subfield containing 1 of the ring of endomorphisms of $\Re_{\Sigma}$. This subfield is isomorphic to $\Sigma$. We shall identify it with $\Sigma$ and we shall write $\sum x_{i}\left(\sigma_{i} \sigma\right)$ as $\left(\sum x_{i} \sigma_{i}\right) \sigma$. Then $\Re_{\Sigma}$ is a vector space over $\Sigma$.

(16) Artin and Whaples [1, pp. 95-97]. 
Consider for the moment $\Re_{\Sigma}$ as a space over the subfield $\Phi$ of $\Sigma$. Thus the endomorphisms of $\Phi$ are the mappings $x=\sum x_{i} \sigma_{i} \rightarrow \sum x_{i}\left(\sigma_{i} \alpha\right), \alpha$ in $\Phi$. Let $\Re_{1}$ denote the totality of vectors $x 1, x \in R, 1 \in \Sigma$. Since $(x 1) \alpha=x(\alpha)=(x \alpha)(1)$, $\Re_{1}$ is a subspace of $\Re_{\Sigma}$ over $\Phi$. This equation and the criterion for equality show that the correspondences $x \rightarrow x 1$ and $\alpha \rightarrow \alpha$ define an isomorphism between the spaces $\Re$ and $\Re_{1}$. We shall now identify $\Re_{1}$ with $\Re$ and write $x 1=x$.

Condition 1 for extension spaces has therefore been verified. The test for equality shows that condition 2 holds. Since any $x=\sum x_{i} \sigma_{i}$ may be written in the form $\sum\left(x_{i}\right) \sigma_{i}, x_{i}$ in $\Re$, condition 3 is also valid and so $\Re_{\Sigma}$ has the required properties.

We suppose now that $\Re^{(1)}$ and $\Re^{(2)}$ are spaces over $\Phi^{(1)}$ and $\Phi^{(2)}$, respectively, and that we have a homomorphism $x^{(1)} \rightarrow x^{(2)}$ of $\Re^{(1)}$ on a subspace $\mathfrak{S}^{(2)}$ of $\Re^{(2)}$ and an isomorphism $\alpha^{(1)} \rightarrow \alpha^{(2)}$ between $\Phi^{(1)}$ and $\Phi^{(2)}$ such that $x^{(1)} \alpha^{(1)} \rightarrow x^{(2)} \alpha^{(2)}$. Furthermore let $\Re_{\Sigma}^{(1)}$ and $\Re_{\Sigma}^{(2)}$ be two spaces over fields $\Sigma^{(1)}$ and $\Sigma^{(2)}$, respectively, satisfying the conditions $1,2,3$. We assume finally that $\Sigma^{(1)}$ and $\Sigma^{(2)}$ are isomorphic under a correspondence $\sigma^{(1)} \rightarrow \sigma^{(2)}$ that is an extension of the correspondence $\alpha^{(1)} \rightarrow \alpha^{(2)}$.

Consider now the correspondence $\sum x_{i}^{(1)} \sigma_{i}^{(1)} \rightarrow \sum x_{i}^{(2)} \sigma_{i}^{(2)}$. We assert that it is single valued. For if $\sum x_{i}^{(1)} \sigma_{i}^{(1)}=\sum y_{j}^{(1)} \tau_{j}^{(1)}$, we may choose vectors $u_{1}^{(1)}, \ldots, u_{r}^{(1)}$ in $\Re^{(1)}$ which are linearly independent over $\Phi^{(1)}$ and write $x_{i}^{(1)}=\sum u_{p}^{(1)} \alpha_{p i}^{(1)}, y_{j}^{(1)}=\sum u_{q}^{(1)} \beta_{g j}^{(1)}$. Then we have $\sum \sum \alpha_{p i}^{(1)} \sigma_{i}^{(1)}=\sum \beta_{p j}^{(1)} \tau_{j}^{(1)}$. Hence $\sum \alpha_{p t}^{(2)} \sigma_{i}^{(2)}=\sum \beta_{p j}^{(2)} \tau_{j}^{(2)}, x_{i}^{(2)}=\sum u_{p}^{(2)} \alpha_{p i}^{(2)}$ and $y_{j}^{(2)}=\sum u_{a}^{(2)} \beta_{a j}^{(2)}$, and these equations imply that $\sum x_{i}^{(2)} \sigma_{i}^{(2)}=\sum y_{j}^{(2)} \sigma_{j}^{(2)}$. This proves the assertion. It is clear that (1) the correspondence $\sum x_{i}^{(1)} \sigma_{i}^{(1)} \rightarrow \sum x_{i}^{(2)} \sigma_{i}^{(2)}$ is a homomorphism, (2) $\left(\sum x_{i}^{(1)} \sigma_{i}^{(1)}\right) \sigma^{(1)}$ $\rightarrow\left(\sum x_{i}^{(2)} \sigma_{i}^{(2)}\right) \sigma^{(2)}$ and (3) the correspondence is an extension of the original correspondence between $\Re^{(1)}$ and $\mathfrak{S}^{(2)}$. It is also clear that there is only one extension of the original correspondence having properties (1) and (2). If the original correspondence is an isomorphism between $\Re^{(1)}$ and $\mathfrak{S}^{(2)}$ the above argument shows that the extension is an isomorphism. If $\mathfrak{S}^{(2)}=\Re^{(2)}$ then the extension maps $\Re_{\Sigma}^{(1)}(1)$ on the whole of $\Re_{\Sigma}^{(2)}(2)$. Hence as a special case of thèse results we see that any two extension spaces of the same space by the same field are isomorphic.

We shall suppose now that $\Re^{(1)}=\Re^{(2)}=\Re, \Phi^{(1)}=\Phi^{(2)}=\Phi, \quad \Sigma^{(1)}=\Sigma^{(2)}=\Sigma$ and let the automorphism in $\Sigma$ be the identity. Then our results may be stated in the following form: If $A$ is a linear transformation in $\Re$ over $\Phi, A$ has a unique extension, which we may also denote as $A$, to a linear transformation in $\Re_{\Sigma}$ over $\Sigma$. If $A$ is (1-1) between $\Re$ and a subspace (the whole space) then its extension is (1-1) between $\Re_{\Sigma}$ and a subspace over $\Sigma$ (the whole space).

These results are all well known in the finite-dimensional case. We recall also that if $(\Re: \Phi)=n$ then $\left(\Re_{\Sigma}: \Sigma\right)=n$, as is clear from 1,2 and 3 .

If $\mathfrak{A}(=\Re)$ is an algebra over $\Phi$, the extension space $\mathfrak{A}_{\Sigma}$ can be made into an algebra by defining $\left(\sum x_{i} \sigma_{i}\right)\left(\sum y_{j} \tau_{j}\right)=\sum\left(x_{i} y_{j}\right)\left(\sigma_{i} \tau_{j}\right)$. It is easy to see that the product thus defined is single valued and distributive, and that the left 
and the right multiplications commute with the endomorphisms $\sigma \in \Sigma$. If $\mathfrak{A}$ is associative (a Lie algebra), then $\mathfrak{A}_{\Sigma}$ is associative (a Lie algebra).

9. Central simple non-associative algebras. We consider now the relation between the multiplication algebra $\mathfrak{M}$ of an arbitrary algebra $\mathfrak{A}$ over a field $\Phi$ and the multiplication algebra of the extension $\mathfrak{A}_{\Sigma}$. As in the case of vector spaces, $\mathfrak{A}$ is a subset of $\mathfrak{A}_{\Sigma}$. If $a \in \mathfrak{A}$, it is evident that the right (left) multiplication $a_{r}\left(a_{l}\right)$ in $\mathfrak{P}_{\Sigma}$ is the extension of the linear transformation $a_{r}\left(a_{l}\right)$ in $\mathfrak{A}$. If $\sum a_{i} \sigma_{i}, a_{i} \in \mathfrak{A}, \sigma_{i} \in \Sigma$, is any element of $\mathfrak{A}_{\Sigma}$, then $\left(\sum a_{i} \sigma_{i}\right)_{r}=\sum\left(a_{i}\right)_{r} \sigma_{i}$, $\left(\sum a_{i} \sigma_{i}\right)_{l}=\sum\left(a_{i}\right)_{l} \sigma_{i}$. It follows that the multiplication algebra of $\mathfrak{A}_{\Sigma}$ is the totality of linear combinations with coefficients in $\Sigma$ of the extensions of the multiplications of $\mathfrak{A}$. Thus $\mathfrak{M}(\mathfrak{A} \Sigma)=\mathfrak{M} \Sigma$ where the $\mathfrak{M}$ on the right-hand side denotes the set of extensions of the multiplications of $\mathfrak{R}$.

We shall call a non-associative algebra $\mathfrak{A}$ over $\Phi$ central if its multiplication centralizer $\mathfrak{E}=\Phi$. If $\mathfrak{A}$ is an arbitrary, nonzero, simple algebra (or ring), we have seen that we may regard $\mathfrak{A}$ as an algebra over its multiplication centralizer. When this is done, $\mathfrak{A}$ becomes a central simple algebra. We may now prove the main theorem on extensions.

THEOREM 17. Any extension $\mathfrak{A}_{\Sigma}$ of a central simple, nonzero, non-associative algebra is central simple.

It suffices to show that $\mathfrak{M}\left(\mathfrak{A}_{\Sigma}\right)$ is a dense set of linear transformations in $\mathfrak{A}_{\Sigma}$ over $\Sigma$. For then $\mathfrak{M}\left(\mathfrak{A}_{\Sigma}\right)$ is irreducible and so $\mathfrak{A}_{\Sigma}$ is simple. Moreover by Theorem 7 , the centralizer of $\mathfrak{M}\left(\mathfrak{U}_{\Sigma}\right)$ is $\Sigma$. Hence the multiplication centralizer $\mathfrak{S}\left(\mathfrak{A}_{\Sigma}\right)=\Sigma$. That $\mathfrak{M}\left(\mathfrak{A}_{\Sigma}\right)$ is dense is an immediate consequence of the above remarks and of the following lemma.

LEMma 3. Let $\mathfrak{A}$ be a dense set of linear transformations in $\Re$ over $\Phi$ and let $\mathfrak{A} \Sigma$ be the totality of $\Sigma$-linear combinations of the extensions of the linear transformations of $\mathfrak{A}$ to linear transformations in $\mathfrak{R}_{\Sigma}$. Then $\mathfrak{A} \Sigma$ is dense in $\mathfrak{R}_{\Sigma}$ over $\Sigma$.

We have to show that if any finite-dimensional subspace $\subseteq$ of $\Re_{\Sigma}$ and any linear transformation $B$ in $\subseteq$ are given, then there is a l.t. $A \in \mathfrak{P} \Sigma$ which coincides with $B$ in $\mathfrak{S}$. Let $y_{1}, y_{2}, \cdots, y_{m}$ be a basis for $\mathfrak{S}$ and let $u_{1}, \cdots, u_{n}$ be vectors of $\Re$ in terms of which all the $y$ 's can be expressed. Then $\subseteq$ is a subspace of the extension $\mathfrak{S}_{0 \Sigma}, \mathfrak{S}_{0}=\left\{u_{1}, \cdots, u_{n}\right\}$. Now there exist 1.t. $E_{i j}, i, j=1, \cdots, n$, in $\mathfrak{A}$ such that $u_{r} E_{i j}=0$ if $r \neq i$ and $u_{i} E_{i j}=u_{j}$. It follows that there is a 1.t. $A$ in $\mathfrak{U} \Sigma$ that induces any l.t. in $\mathfrak{S}_{0 \Sigma}$. Hence there is an $A$ that induces the linear transformation $B$ in the subspace $\mathfrak{S}$ of $\mathfrak{S}_{0}$.

\section{SPECIAL ALgebras of linear transformations}

10. Linear transformations in vector spaces over a field. In this part we shall be concerned mainly with dense algebras of finite valued linear trans-

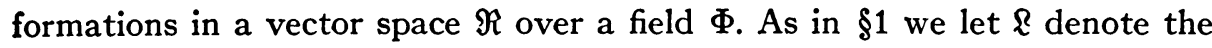


complete set of 1.t. and let $\mathfrak{F}$ be the subset of finite valued 1.t. Both $\mathfrak{R}$ and $\mathfrak{F}$ are algebras over $\Phi$, the set of multiplications by the elements of the underlying field $\Phi$. We have seen that if $\mathfrak{A}$ is a dense subalgebra of $\mathfrak{F}$, then $\mathfrak{A}$ is simple and $\mathfrak{A}$ contains minimal right ideals. We prove next that $\mathfrak{A}$ is central by proving the following lemma.

LEMMA 4. If $\mathfrak{A}$ is a dense ring of linear transformations in a vector space $\mathfrak{R}$ over a field $\Phi$, then the transformations of the multiplication centralizer $\mathfrak{S}$ of $\mathfrak{A}$ are multiplications by the elements of $\Phi$.

In order to avoid confusion we write the effect of $\gamma \in \mathfrak{C}$ on $X \in \mathfrak{A}$ as $X^{\gamma}$. Then we have $(X Y)^{r}=X^{r} Y=X\left(Y^{r}\right)$. We choose a vector $u \neq 0$ in $\Re$ and suppose that $X$ is a 1.t. in $\mathfrak{A}$ such that $u X=0$. Then $u X^{r}=0$ also. For otherwise there is a $Y \in \mathfrak{A}$ such that $\left(u X^{\gamma}\right) Y \neq 0$ contrary to $u X^{\gamma} Y=(u X) Y^{\gamma}=0$. It follows from this that the mapping $v=u X \rightarrow u X^{\gamma}=v^{\prime}$ is single valued and defined in the whole of $\Re$. It is clear that $v \rightarrow v^{\prime}$ is an endomorphism and since $(u X) A \rightarrow u(X A)^{r}=\left(u X^{\gamma}\right) A, v \rightarrow v^{\prime}$ is in the centralizer of $\mathfrak{A}$. Hence by Theorem 7 there is a $\gamma \in \Phi$ such that $u X^{\gamma}=u(X \gamma)$ for all $X \in \mathfrak{A}$. Replacing $X$ by $A X$ yields the equation $u(A X)^{\gamma}=u(A X \gamma)$ so that $(u A) X^{\gamma}=(u A)(X \gamma)$. As $A$ ranges over $\mathfrak{A}, u A$ ranges over $\Re$. Hence $X^{\gamma}=X \gamma$.

If $\mathfrak{A}$ is an algebra, $\mathfrak{E} \geqq \Phi$. Hence if $\mathfrak{A}$ is a dense algebra, $\mathfrak{E}=\Phi$ and $\mathfrak{A}$ is central.

We shall call an algebra $\mathfrak{A}$ over $\Phi$ algebraic if every element of $\mathfrak{A}$ satisfies some non-trivial polynomial equation, or what is the same thing, if every element generates an algebra of finite dimensionality. We shall call $\mathfrak{A}$ locally finite if every finite subset of $\mathfrak{A}$ generates a finite-dimensional subalgebra. It is an open question whether or not simple algebras that are algebraic are necessarily locally finite. We shall show next that any dense algebra of finite valued 1.t. in $\Re$ over $\Phi$ is locally finite. For this purpose we require the following lemma.

Lemma 5. If $A_{1}, A_{2}, \cdots, A_{n}$ are finite valued linear transformations in $\mathfrak{R}$, there exists a decomposition $\Re=\mathfrak{S} \oplus \mathfrak{N}$ where $\subseteq$ is a finite-dimensional subspace invariant under all the $A_{i}$ and $\mathfrak{N}$ is annihilated by all the $A_{i}$.

We shall show first that if $\Re_{1}$ is any subspace of $\Re, \Re_{1}=\mathfrak{B}_{1} \oplus \mathfrak{M}_{1}$ where $\mathfrak{B}_{1} A_{1}=0$ and $\mathfrak{M}_{1}$ is finite-dimensional. Now $\Re_{1} A_{1}$ is finite-dimensional so that $\Re_{1} A_{1}=\left\{y_{1}, \cdots, y_{r}\right\}$ where the $y_{i}$ are linearly independent. We may choose vectors $x_{i} \in \Re_{1}$ such that $x_{i} A_{1}=y_{i}$ and we set $\mathfrak{M}_{1}=\left\{x_{1}, \cdots, x_{r}\right\}$. Then if $x$ is any vector in $\Re_{1}, x A_{1}=\sum y_{i} \alpha_{i}=\left(\sum x_{i} \alpha_{i}\right) A_{1}$. Hence if $z=x-\sum x_{i} \alpha_{i}, z A_{1}=0$ and $x=z+\sum x_{i} \alpha_{i} \in \mathfrak{Z}_{1}+\mathfrak{M}_{1}$ where $\mathfrak{B}_{1}$ is the subspace of $\Re_{1}$ of vectors annihilated by $A_{1}$. If $\left(\sum x_{i} \alpha_{i}\right) A_{1}=0, \sum y_{i} \alpha_{i}=0$ and all the $\alpha_{i}=0$. This shows that $\mathfrak{Z}_{1} \wedge \mathfrak{M}_{1}=0$ and so $\Re_{1}=\mathfrak{B}_{1} \oplus \mathfrak{M}_{1}$. We now begin with $\Re_{1}=\Re$ and write $\Re=\mathfrak{Z}_{1} \oplus \mathfrak{M}_{1}$, where $\mathfrak{Z}_{1} A_{1}=0$ and $\mathfrak{M}_{1}$ is finite-dimensional. We then repeat the argument with $\mathfrak{B}_{1}$ and $A_{2}$ and obtain $\mathfrak{B}_{1}=\mathfrak{Z}_{2} \oplus \mathfrak{M}_{2}$ where $\mathfrak{B}_{2} A_{1}=\mathfrak{B}_{2} A_{2}=0$ and 
$\mathfrak{M}_{2}$ is finite-dimensional. Then $\Re=\mathfrak{Z}_{2} \oplus \mathfrak{M}_{1} \oplus \mathfrak{M}_{2}$. Continuing in this way we obtain finally that $\Re=3 \oplus \mathfrak{M}$ where $3 A_{i}=0, i=1, \cdots, n$, and $\mathfrak{M}$ is finitedimensional. Since $3 A_{i}=0$, we have $\mathfrak{R} A_{i}=\mathfrak{M} A_{i}$. Hence $\mathfrak{S}=\mathfrak{M}+\mathfrak{M} A_{1}+\ldots$ $+\mathfrak{M} A_{n}$ is a finite-dimensional subspace invariant under all the $A_{i}$. We have $\mathfrak{S}=\mathfrak{Z}^{\prime} \oplus \mathfrak{M}$ where $\mathfrak{Z}^{\prime}=\subseteq \wedge \mathfrak{B}$ and we may decompose $\mathbb{B}$ as $\mathbb{B}=\mathfrak{Z}^{\prime} \oplus \mathfrak{R}$. Then $\Re=S \oplus \mathfrak{S}$ where $\mathfrak{R} A_{i}=0$ as required.

Now by Lemma 5 we see that the linear transformations $A_{1}, \cdots, A_{n}$ generate an algebra which is isomorphic to an algebra of linear transormations in a finite-dimensional space $\mathfrak{S}$. Hence the algebra generated by the $A_{i}$ is finite-dimensional over $\Phi$. We summarize our results in the following theorem:

THEOREM 18. Let $\Re$ be a vector space over a field $\Phi$ and let $\mathfrak{A}$ be a dense algebra of finite valued linear transformations in $\Re$ over $\Phi$. Then $\mathfrak{A}$ is central simple and locally finite.

11. Isomorphisms and automorphisms of algebras of linear transformations. Let $\mathfrak{A}_{1}$ and $\mathfrak{A}_{2}$ be two dense algebras in $\Re$ over $\Phi$ which are isomorphic under a correspondence $A_{1} \rightarrow A_{2}$. By an isomorphism here we mean an algebra isomorphism, that is, $A_{1} \alpha \rightarrow A_{2} \alpha$ for all $\alpha$ in $\Phi$. By Theorem 12 there is a semilinear transformation $S$ in $\Re$ over $\Phi$ such that $A_{2}=S^{-1} A_{1} S$. If $\alpha \rightarrow \bar{\alpha}$ is the automorphism in $\Phi$ associated with $\subseteq$ we have $\bar{\alpha}=S^{-1} \alpha S$. Since $S^{-1}\left(A_{1} \alpha\right) S=A_{2} \alpha$ it follows that $\bar{\alpha}=\alpha$ and hence $S$ is a linear transformation. This theorem may be stated in the following form:

THEOREM 19. If $\mathfrak{A}_{1}$ and $\mathfrak{A}_{2}$ are isomorphic dense algebras of linear transformations in $\Re$ over $\Phi$ any isomorphism between $\mathfrak{A}_{1}$ and $\mathfrak{A}_{2}$ can be extended to an inner automorphism in $\mathfrak{R}$, the complete algebra of linear transformations.

This, of course, gives the following corollary.

CoRollaRY. If $\mathfrak{A}$ is a dense subalgebra of $\mathfrak{R}$, any automorphism in $\mathfrak{A}$ can be extended to an inner automorphism in $\mathbb{R}$.

We may now prove the following theorem.

TheOREM 20. Any automorphism in $\mathfrak{R}$ is inner.

We remark first that if $X \rightarrow X^{G}$ is an automorphism in $\mathfrak{R}$, then $\mathfrak{F}^{G}=\mathfrak{F}$. This is clear since $\mathfrak{F}$, and hence $\mathfrak{F}^{\sigma}$, is contained in every two-sided ideal not equal to 0 in $\mathfrak{R}$. Thus $G$ induces an automorphism in $\mathfrak{F}$. Hence there is an $S$ in $\mathfrak{R}$ such that $F^{G}=S^{-1} F S$ for all $F$ in $\mathfrak{F}$. Now consider the automorphism $X \rightarrow X^{B}=S X^{\sigma} S^{-1}$. We have $F^{B}=F$ for all $F$ in $\mathfrak{F}$. If $x$ is any vector there is an $F$ in $\mathfrak{F}$ such that $x F=x$. Hence $x X=x F X=x(F X)^{B}=x F^{H} X^{H}=x X^{H}$ and so $X^{H}=X$. This proves that $H$ is the identity and $G$ is the inner automorphism $X \rightarrow S^{-1} X S$. 
B. H. ARNOLd

\section{BIBLIOGRAPHY}

1. Rings of operators on vector spaces, Ann. of Math. vol. 45 (1944) pp. 24-49.

E. Artin and G. Whaples

1. The theory of simple rings, Amer. J. Math. vol. 65 (1943) pp. 87-107.

H. Firting

1. Primarkomponentenzerlegung in nichtkommutativen Ringe, Math. Ann. vol. 111 (1935) pp. 19-41.

N. JACOBSON

1. The theory of rings, Mathematical Surveys, vol. 2, New York, 1943.

2. A note on non-associative algebras, Duke Math. J. vol. 3 (1937) pp. 544-548.

3. Pseudo-linear transformations, Ann. of Math. vol. 38 (1937) pp. 484-507.

E. NOETHER

1. Nichtkommutativ Algebra, Math. Zeit. vol. 37 (1933) pp. 514-541.

O. ORE

1. Formale Theorie lineare Differentialgleichungen. II. Journal für Mathematik vol. 168 (1932) pp. 233-252.

The Johns Hopkins University,

BALTIMORE, MD. 